Acta Crystallographica Section E

Structure Reports

Online

ISSN 1600-5368

\section{Ethyl anthracene-9-carboxylate}

\section{Edwin Weber, ${ }^{\text {a }}$ Wilhelm Seichter, ${ }^{\text {a }}$ Conrad Fischer, ${ }^{\text {a }}$ L. M. S. Franziska Bendrath ${ }^{a}$ and Bakhtiyar T. Ibragimov ${ }^{b_{*}}$}

anstitut für Organische Chemie, TU Bergakademie Freiberg, Leipziger Strasse 29, D-09596 Freiberg/Sachsen, Germany, and ${ }^{\mathbf{b}}$ Institute of Bioorganic Chemistry, Academy of Sciences of Uzbekistan, H Abdullaev 83, Tashkent 100125, Uzbekistan Correspondence e-mail: bahtier@academy.uzsci.net

Received 28 April 2008; accepted 12 June 2008

Key indicators: single-crystal X-ray study; $T=153 \mathrm{~K}$; mean $\sigma(\mathrm{C}-\mathrm{C})=0.002 \AA$; $R$ factor $=0.035 ; w R$ factor $=0.085 ;$ data-to-parameter ratio $=11.6$.

In the title compound, $\mathrm{C}_{17} \mathrm{H}_{14} \mathrm{O}_{2}$, the $\mathrm{COO}$ group and the anthracene fragment form a dihedral angle of $76.00(19)^{\circ}$. The torsion angle around the $\mathrm{O}-\mathrm{Csp}^{3}$ bond of the ester group is $108.52(18)^{\circ}$. The crystal structure is stabilized by $\mathrm{C}-\mathrm{H} \cdots \mathrm{O}$ interactions and edge-to-face arene interactions with $\mathrm{C}-$ $\mathrm{H}$. . (ring centroid) distances in the range $2.75-2.84 \AA$.

\section{Related literature}

For related crystal structures, see: Bart \& Schmidt (1971); Heller \& Schmidt (1971); Sweeting et al. (1997). For the preparation of the title compound, see: Larsen \& Harpp (1980).<smiles>CCOC(=O)c1c2ccccc2cc2ccccc12</smiles>

\section{Experimental}

Crystal data

$\mathrm{C}_{17} \mathrm{H}_{14} \mathrm{O}_{2}$

$M_{r}=250.28$

$$
\begin{aligned}
& b=10.2137(7) \AA \\
& c=14.5426(11) \AA \\
& V=1268.94(16) \AA^{3} \\
& Z=4
\end{aligned}
$$

$$
\begin{aligned}
& \text { Mo } K \alpha \text { radiation } \\
& \mu=0.09 \mathrm{~mm}^{-1} \\
& T=153(2) \mathrm{K} \\
& 0.25 \times 0.25 \times 0.20 \mathrm{~mm}
\end{aligned}
$$

Data collection

Bruker Kappa APEXII CCD diffractometer

Absorption correction: none

15373 measured reflections

Refinement

$R\left[F^{2}>2 \sigma\left(F^{2}\right)\right]=0.035$

$w R\left(F^{2}\right)=0.085$

$S=1.04$

2020 reflections

174 parameters

2020 independent reflections 1600 reflections with $I>2 \sigma(I)$ $R_{\text {int }}=0.047$

\section{Table 1}

Hydrogen-bond geometry $\left(\AA{ }^{\circ}\right)$.

\begin{tabular}{lllll}
\hline$D-\mathrm{H} \cdots A$ & $D-\mathrm{H}$ & $\mathrm{H} \cdots A$ & $D \cdots A$ & $D-\mathrm{H} \cdots A$ \\
\hline $\mathrm{C} 5-\mathrm{H} 5 \cdots \mathrm{O} 1^{\mathrm{i}}$ & 0.93 & 2.53 & $3.302(2)$ & 140 \\
\hline Symmetry code: (i) $-x,-y+2, z+\frac{1}{2}$. &
\end{tabular}

Data collection: APEX2 (Bruker, 2004); cell refinement: SAINT (Bruker, 2004); data reduction: $S A I N T$; program(s) used to solve structure: SHELXS97 (Sheldrick, 2008); program(s) used to refine structure: SHELXL97 (Sheldrick, 2008); molecular graphics: ORTEP-3 (Farrugia, 1997); software used to prepare material for publication: SHELXTL (Sheldrick, 2008).

Financial support from the German Federal Ministry of Economics and Technology (BMWi) under grant No. 16IN0218 'ChemoChips' is gratefully acknowledged. L. M. S. F. Bendrath thanks Dr T. Gruber for supervision of her practical work.

Supplementary data and figures for this paper are available from the IUCr electronic archives (Reference: GK2145).

\section{References}

Bart, J. C. J. \& Schmidt, J. (1971). Isr. J. Chem. 9, 429-448.

Bruker (2004). APEX2 and SAINT. Bruker AXS Inc., Madison, Wisconsin, USA.

Farrugia, L. J. (1997). J. Appl. Cryst. 30, 565.

Heller, E. \& Schmidt, J. (1971). Isr. J. Chem. 9, 449-462.

Larsen, C. \& Harpp, D. N. (1980). J. Org. Chem. 45, 3713-3716.

Sheldrick, G. M. (2008). Acta Cryst. A64, 112-122.

Sweeting, L. M., Rheingold, A. L., Gingerich, J. M., Rutter, A. W., Spence, R. A., Cox, C. D. \& Kim, T. J. (1997). Chem. Mater. 9, 1103-1115. 


\section{supporting information}

Acta Cryst. (2008). E64, o1288 [doi:10.1107/S1600536808017819]

\section{Ethyl anthracene-9-carboxylate}

\section{Edwin Weber, Wilhelm Seichter, Conrad Fischer, L. M. S. Franziska Bendrath and Bakhtiyar T. Ibragimov}

\section{S1. Comment}

9-Anthracenecarboxylic acid esters are of current interest in materials science (Sweeting et al., 1997). The conformational features of the title compound (Fig. 1) resemble those found in the crystal structure of the analogous methyl 9-anthracenecarboxylate (Bart \& Schmidt, 1971). A comparative examination of the crystal structures, however, reveals that a slight modification of the molecular structure has a fundamental influence on the molecular packing mode. According to the presence of a twofold screw axis, helical hydrogen bonded strands (Table 1, Fig. 2) running along the $c$ axis are the basic supramolecular entities of the present crystal structure. Furthermore, the anthracene units of neighbouring strands are arranged in "edge-to-face" herringbone fashion with the closest intermolecular distance of 2.86 $\AA$.

\section{S2. Experimental}

9-Anthracenecarbonyl chloride (300 $\mathrm{mg})$ in $\mathrm{CH}_{2} \mathrm{Cl}_{2}(45 \mathrm{ml})$ was reacted with ethanol $(10 \mathrm{ml})$ and pyridine $(2 \mathrm{ml})$. The resulting solution was heated under reflux for $11 \mathrm{~h}$, then cooled to room temperature and subsequently extracted three times with $2 \mathrm{~N}$ aqueous $\mathrm{HCl}$ and water $\left(50 \mathrm{ml}\right.$, each), and finally two times with water $(100 \mathrm{ml})$. After addition of $\mathrm{CH}_{2} \mathrm{Cl}_{2}$ (200 $\mathrm{ml}$ ) the organic layer was dried over $\mathrm{CaCl}_{2}$ and the solvent removed under reduced pressure. Recrystallization of the white powder from acetone yielded colourless crystals suitable for X-ray diffraction analysis. (82\%, m.p. 381-382 K). Anal. Calcd. for $\mathrm{C}_{17} \mathrm{H}_{14} \mathrm{O}_{2}$ : C 81.58; H 5.64; Found: C 81.42; H 5.90\%.

\section{S3. Refinement}

In absence of significant anomalous scattering effects, Friedel pairs were merged prior to refinement. All hydrogen atoms were positioned geometrically and refined using the riding model with $\mathrm{d}(\mathrm{C}-\mathrm{H})=0.93 \AA, U_{\text {iso }}=1.2 \mathrm{Ueq}(\mathrm{C})$ for aromatic, $0.96 \AA, U_{\text {iso }}=1.5 \mathrm{Ueq}(\mathrm{C})$ for $\mathrm{CH}_{3}$ and $0.97 \AA, U_{\text {iso }}=1.2 \mathrm{Ueq}(\mathrm{C})$ for $\mathrm{CH}_{2} \mathrm{H}$ atoms. 


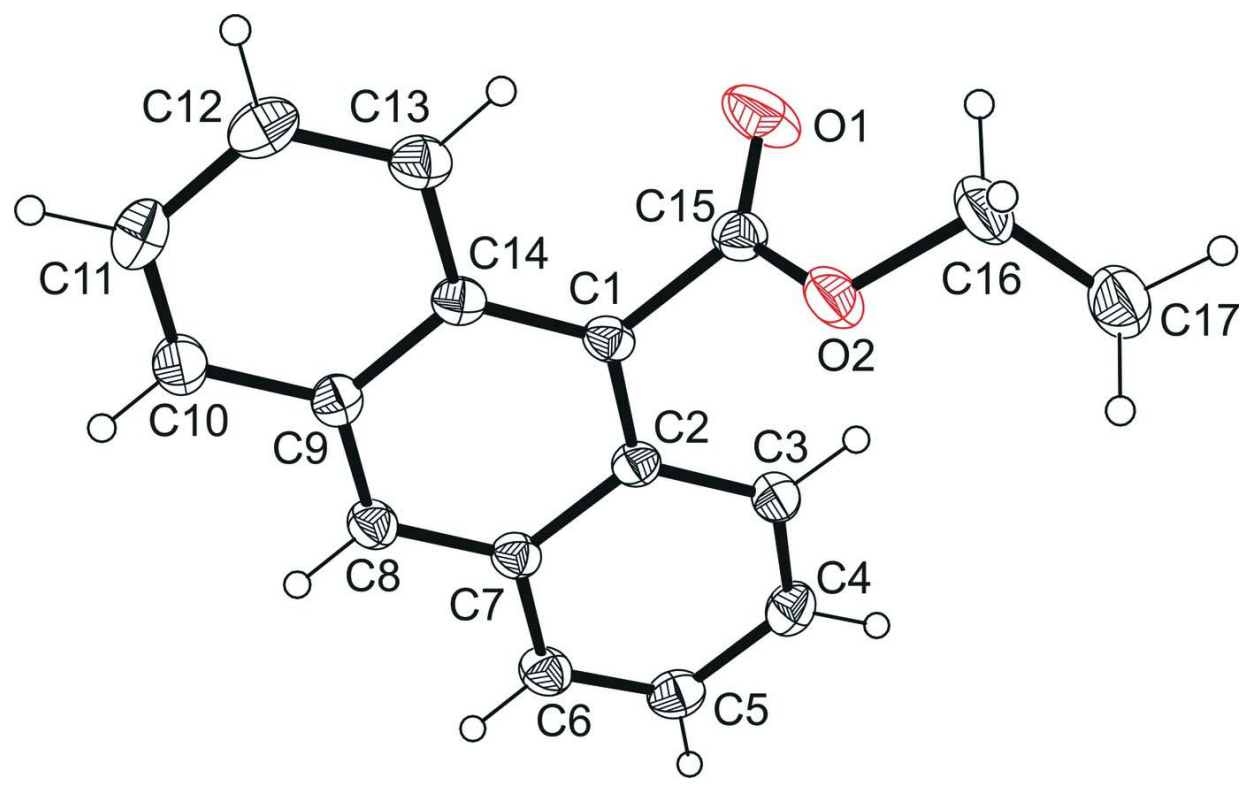

\section{Figure 1}

Molecular structure of the title compound with atomic labels and 50\% probability displacement ellipsoids for non $\mathrm{H}$ atoms.

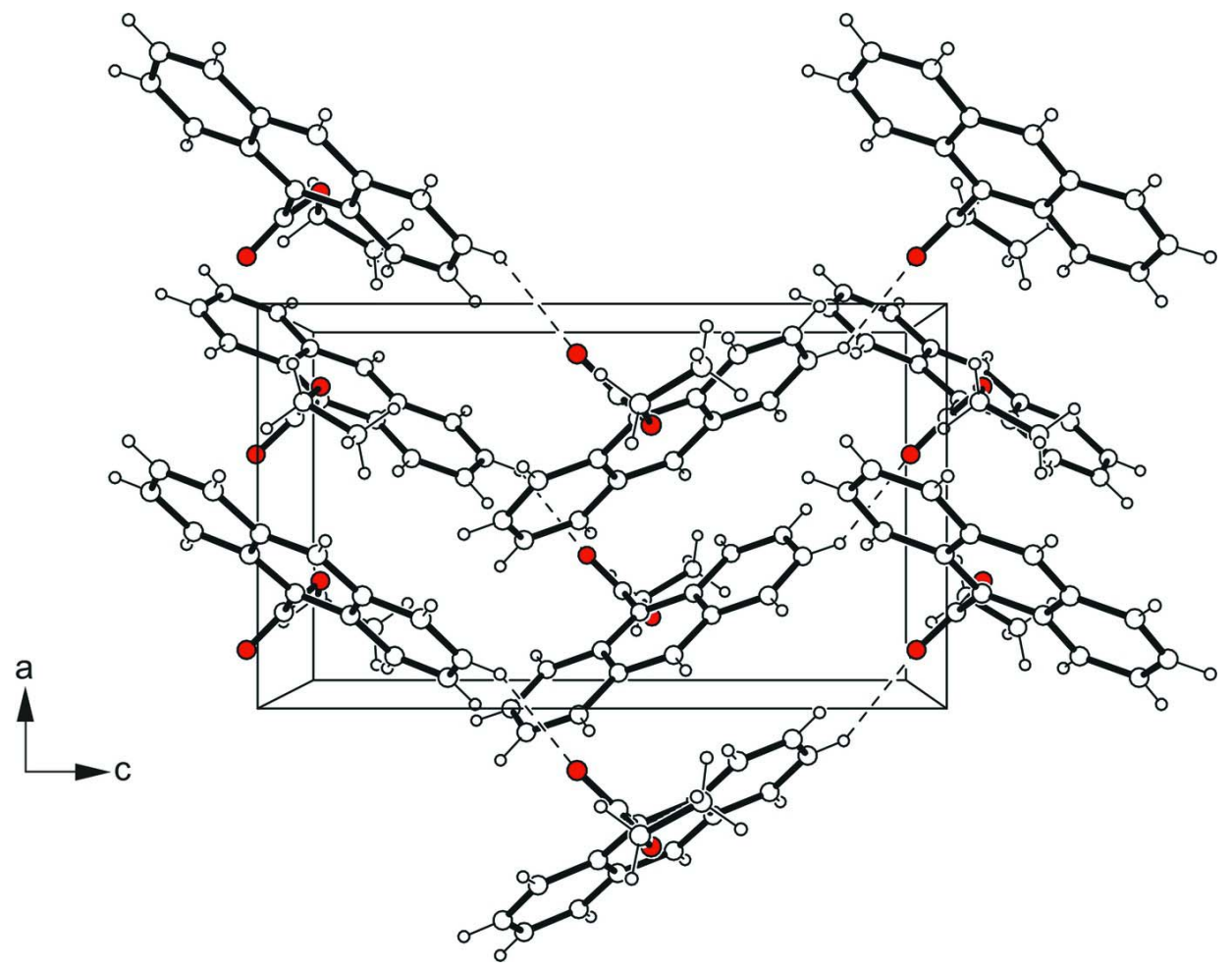

\section{Figure 2}

Crystal packing of the title compound viewed along the $b$ axis. 


\section{Ethyl anthracene-9-carboxylate}

Crystal data

$$
\mathrm{C}_{17} \mathrm{H}_{14} \mathrm{O}_{2}
$$

$M_{r}=250.28$

Orthorhombic, Pna2 $_{1}$

Hall symbol: P 2c -2n

$a=8.5431(6) \AA$

$b=10.2137$ (7) $\AA$

$c=14.5426(11) \AA$

$V=1268.94(16) \AA^{3}$

$Z=4$

\section{Data collection}

\section{Bruker Kappa APEXII CCD} diffractometer

Radiation source: fine-focus sealed tube

Graphite monochromator

$\varphi$ and $\omega$ scans

15373 measured reflections

2020 independent reflections

\section{Refinement}

Refinement on $F^{2}$

Least-squares matrix: full

$R\left[F^{2}>2 \sigma\left(F^{2}\right)\right]=0.035$

$w R\left(F^{2}\right)=0.085$

$S=1.04$

2020 reflections

174 parameters

1 restraint

Primary atom site location: structure-invariant direct methods

\author{
$F(000)=528$ \\ $D_{\mathrm{x}}=1.310 \mathrm{Mg} \mathrm{m}^{-3}$ \\ Mo $K \alpha$ radiation, $\lambda=0.71073 \AA$ \\ Cell parameters from 4881 reflections \\ $\theta=2.4-30.5^{\circ}$ \\ $\mu=0.09 \mathrm{~mm}^{-1}$ \\ $T=153 \mathrm{~K}$ \\ Irregular, colourless \\ $0.25 \times 0.25 \times 0.20 \mathrm{~mm}$
}

1600 reflections with $I>2 \sigma(I)$

$R_{\text {int }}=0.047$

$\theta_{\max }=30.6^{\circ}, \theta_{\min }=2.4^{\circ}$

$h=-11 \rightarrow 12$

$k=-12 \rightarrow 14$

$l=-20 \rightarrow 13$

Secondary atom site location: difference Fourier map

Hydrogen site location: inferred from neighbouring sites

$\mathrm{H}$-atom parameters constrained

$w=1 /\left[\sigma^{2}\left(F_{\mathrm{o}}^{2}\right)+(0.0459 P)^{2}\right]$

where $P=\left(F_{\mathrm{o}}^{2}+2 F_{\mathrm{c}}{ }^{2}\right) / 3$

$(\Delta / \sigma)_{\max }<0.001$

$\Delta \rho_{\max }=0.22$ e $\AA^{-3}$

$\Delta \rho_{\min }=-0.17 \mathrm{e} \AA^{-3}$

\section{Special details}

Experimental. ${ }^{1} \mathrm{H}-\mathrm{NMR}\left(400 \mathrm{MHz}, \mathrm{CDCl}_{3}\right.$, $\delta$, p.p.m.): $1.53\left(\mathrm{~m}, \mathrm{CH}_{3}\right) ; 4.68\left(\mathrm{q},{ }^{3} \mathrm{~J}=7.2 \mathrm{~Hz}, \mathrm{OCH}_{2}, 2 \mathrm{H}\right) ; 7.45\left(\mathrm{~m}, \mathrm{H}_{2}, \mathrm{H}_{3}\right.$, $\left.\mathrm{H}_{6}, \mathrm{H}_{7}, 4 \mathrm{H}\right) ; 8.03$ (t, $\left.\mathrm{H}_{1}, \mathrm{H}_{4}, \mathrm{H}_{5}, \mathrm{H}_{8}, 4 \mathrm{H}\right) ; 8.54$ (t, $\left.\mathrm{H}_{10}, 1 \mathrm{H}\right) .{ }^{13} \mathrm{C}-\mathrm{NMR}\left(100 \mathrm{MHz}, \mathrm{CDCl}_{3}, \delta\right.$, p.p.m.): $13.70\left(\mathrm{CH}_{3}\right), 61.70$ $\left(\mathrm{OCH}_{2}\right), 125.17\left(\mathrm{C}_{1}, \mathrm{C}_{8}\right), 125.86\left(\mathrm{C}_{3}, \mathrm{C}_{6}\right), 127.25\left(\mathrm{C}_{2}, \mathrm{C}_{7}\right) ; 128.37\left(\mathrm{C}_{9}, \mathrm{C}_{4 \mathrm{a}}, \mathrm{C}_{10 a}\right) ; 128.89\left(\mathrm{C}_{4}, \mathrm{C}_{5}\right) ; 129.23\left(\mathrm{C}_{10}\right) ; 131.35$ $\left(\mathrm{C}_{8 \mathrm{a}}, \mathrm{C}_{9 \mathrm{a}}\right) ; 169.10(\mathrm{CO})$. IR $\left(\mathrm{KBr}, \mathrm{cm}^{-1}\right): 3079(\mathrm{w}), 3053(\mathrm{w})\left(\mathrm{C}-\mathrm{H}_{\mathrm{ar}}\right) ; 2981(\mathrm{~m}), 2929,2904,2867(\mathrm{C}-\mathrm{H}) ; 1952 ; 1802$; 1715 (C?O); 1626; 1564; 1522; 1467; 1455; 1420; 1388; 1372; 1352; 1321; 1288; 1264; 1238; 1216; 1171; 1151; 1119; 1099; 1025; 974; 957; 935; 897; 866; 846; 810; 740; 671; 633; 607; 560; 529; 452. GC-MS m/z $250\left(100, M^{+}\right), 235$, $222,205,177,151,139,126,102,88,75,51$.

Geometry. All e.s.d.'s (except the e.s.d. in the dihedral angle between two 1.s. planes) are estimated using the full covariance matrix. The cell e.s.d.'s are taken into account individually in the estimation of e.s.d.'s in distances, angles and torsion angles; correlations between e.s.d.'s in cell parameters are only used when they are defined by crystal symmetry. An approximate (isotropic) treatment of cell e.s.d.'s is used for estimating e.s.d.'s involving l.s. planes.

Refinement. Refinement of $F^{2}$ against ALL reflections. The weighted $R$-factor $w R$ and goodness of fit $S$ are based on $F^{2}$, conventional $R$-factors $R$ are based on $F$, with $F$ set to zero for negative $F^{2}$. The threshold expression of $F^{2}>\sigma\left(F^{2}\right)$ is used only for calculating $R$-factors(gt) $e t c$. and is not relevant to the choice of reflections for refinement. $R$-factors based on $F^{2}$ are statistically about twice as large as those based on $F$, and $R$ - factors based on ALL data will be even larger. 
Fractional atomic coordinates and isotropic or equivalent isotropic displacement parameters $\left(\AA^{2}\right)$

\begin{tabular}{|c|c|c|c|c|}
\hline & $x$ & $y$ & $z$ & $U_{\text {iso }} * / U_{\text {eq }}$ \\
\hline $\mathrm{O} 1$ & $0.13453(17)$ & $0.82389(13)$ & $-0.03306(11)$ & $0.0430(4)$ \\
\hline $\mathrm{O} 2$ & $0.30770(15)$ & $0.75339(12)$ & $0.07149(10)$ & $0.0316(3)$ \\
\hline $\mathrm{C} 1$ & $0.28393(19)$ & $0.97928(15)$ & $0.05261(11)$ & $0.0195(3)$ \\
\hline $\mathrm{C} 2$ & $0.23153(19)$ & $1.03155(15)$ & $0.13680(11)$ & $0.0198(3)$ \\
\hline $\mathrm{C} 3$ & $0.1270(2)$ & $0.96373(17)$ & $0.19647(11)$ & $0.0233(4)$ \\
\hline $\mathrm{H} 3$ & 0.0919 & 0.8805 & 0.1806 & $0.028^{*}$ \\
\hline $\mathrm{C} 4$ & $0.0777(2)$ & $1.01972(17)$ & $0.27676(12)$ & $0.0264(4)$ \\
\hline $\mathrm{H} 4$ & 0.0082 & 0.9747 & 0.3145 & $0.032 *$ \\
\hline $\mathrm{C} 5$ & $0.1310(2)$ & $1.14542(18)$ & $0.30335(12)$ & 0.0269 (4) \\
\hline H5 & 0.0973 & 1.1818 & 0.3585 & $0.032 *$ \\
\hline C6 & $0.2313(2)$ & $1.21299(17)$ & $0.24865(12)$ & $0.0249(4)$ \\
\hline H6 & 0.2662 & 1.2951 & 0.2672 & $0.030 *$ \\
\hline $\mathrm{C} 7$ & $0.2840(2)$ & $1.16012(15)$ & $0.16313(11)$ & $0.0206(3)$ \\
\hline $\mathrm{C} 8$ & $0.3840(2)$ & $1.22952(16)$ & $0.10477(12)$ & $0.0222(3)$ \\
\hline $\mathrm{H} 8$ & 0.4185 & 1.3121 & 0.1226 & $0.027^{*}$ \\
\hline C9 & $0.4334(2)$ & $1.17882(15)$ & $0.02076(11)$ & $0.0210(3)$ \\
\hline $\mathrm{C} 10$ & $0.5365(2)$ & $1.24932(16)$ & $-0.03850(12)$ & $0.0269(4)$ \\
\hline $\mathrm{H} 10$ & 0.5701 & 1.3325 & -0.0216 & $0.032 *$ \\
\hline C11 & $0.5865(2)$ & $1.19688(18)$ & $-0.11930(14)$ & $0.0307(4)$ \\
\hline H11 & 0.6544 & 1.2441 & -0.1567 & $0.037 *$ \\
\hline $\mathrm{C} 12$ & $0.5355(2)$ & $1.07060(19)$ & $-0.14688(12)$ & $0.0294(4)$ \\
\hline H12 & 0.5700 & 1.0358 & -0.2024 & $0.035^{*}$ \\
\hline $\mathrm{C} 13$ & $0.4366(2)$ & $1.00008(16)$ & $-0.09291(11)$ & $0.0256(4)$ \\
\hline H13 & 0.4040 & 0.9176 & -0.1122 & $0.031 *$ \\
\hline C14 & $0.38172(19)$ & $1.05069(16)$ & $-0.00681(11)$ & $0.0201(3)$ \\
\hline $\mathrm{C} 15$ & $0.2313(2)$ & $0.84551(16)$ & $0.02446(12)$ & $0.0224(3)$ \\
\hline $\mathrm{C} 16$ & $0.2630(2)$ & $0.61657(16)$ & $0.05647(15)$ & $0.0336(4)$ \\
\hline H16A & 0.3556 & 0.5640 & 0.0454 & $0.040 *$ \\
\hline H16B & 0.1954 & 0.6100 & 0.0031 & $0.040^{*}$ \\
\hline C17 & $0.1792(2)$ & 0.56759 (19) & $0.13964(15)$ & $0.0366(5)$ \\
\hline H17A & 0.2438 & 0.5802 & 0.1929 & $0.055^{*}$ \\
\hline H17B & 0.1569 & 0.4760 & 0.1324 & $0.055^{*}$ \\
\hline $\mathrm{H} 17 \mathrm{C}$ & 0.0830 & 0.6150 & 0.1471 & $0.055^{*}$ \\
\hline
\end{tabular}

Atomic displacement parameters $\left(\AA^{2}\right)$

\begin{tabular}{lllllll}
\hline & $U^{11}$ & $U^{22}$ & $U^{33}$ & $U^{12}$ & $U^{13}$ & $U^{23}$ \\
\hline O1 & $0.0469(9)$ & $0.0323(8)$ & $0.0498(9)$ & $-0.0006(6)$ & $-0.0262(8)$ & $-0.0080(6)$ \\
O2 & $0.0389(8)$ & $0.0185(5)$ & $0.0374(7)$ & $-0.0030(5)$ & $-0.0137(6)$ & $-0.0007(5)$ \\
C1 & $0.0205(8)$ & $0.0176(7)$ & $0.0204(7)$ & $0.0016(6)$ & $-0.0040(6)$ & $-0.0016(6)$ \\
C2 & $0.0201(8)$ & $0.0202(7)$ & $0.0192(7)$ & $0.0007(6)$ & $-0.0040(6)$ & $0.0016(6)$ \\
C3 & $0.0239(9)$ & $0.0218(8)$ & $0.0243(9)$ & $-0.0021(6)$ & $-0.0015(7)$ & $0.0004(6)$ \\
C4 & $0.0260(9)$ & $0.0292(9)$ & $0.0239(8)$ & $-0.0010(7)$ & $0.0038(7)$ & $0.0037(7)$ \\
C5 & $0.0303(10)$ & $0.0298(9)$ & $0.0204(8)$ & $0.0032(7)$ & $0.0022(7)$ & $-0.0041(7)$ \\
C6 & $0.0293(10)$ & $0.0210(8)$ & $0.0245(8)$ & $0.0005(7)$ & $-0.0007(8)$ & $-0.0047(7)$
\end{tabular}


supporting information

\begin{tabular}{lllllll} 
C7 & $0.0220(9)$ & $0.0190(7)$ & $0.0208(7)$ & $0.0012(6)$ & $-0.0019(6)$ & $-0.0014(6)$ \\
C8 & $0.0241(9)$ & $0.0190(8)$ & $0.0235(8)$ & $-0.0008(6)$ & $-0.0015(7)$ & $-0.0016(6)$ \\
C9 & $0.0203(8)$ & $0.0203(7)$ & $0.0225(7)$ & $0.0015(6)$ & $-0.0011(7)$ & $0.0012(6)$ \\
C10 & $0.0262(10)$ & $0.0257(8)$ & $0.0289(9)$ & $-0.0027(7)$ & $-0.0001(7)$ & $0.0017(7)$ \\
C11 & $0.0293(10)$ & $0.0337(10)$ & $0.0292(9)$ & $-0.0003(8)$ & $0.0066(8)$ & $0.0068(8)$ \\
C12 & $0.0298(10)$ & $0.0361(10)$ & $0.0222(8)$ & $0.0067(8)$ & $0.0039(7)$ & $-0.0006(7)$ \\
C13 & $0.0286(10)$ & $0.0246(8)$ & $0.0235(8)$ & $0.0021(7)$ & $-0.0007(7)$ & $-0.0039(6)$ \\
C14 & $0.0198(8)$ & $0.0206(7)$ & $0.0200(8)$ & $0.0025(6)$ & $-0.0021(6)$ & $-0.0014(6)$ \\
C15 & $0.0232(9)$ & $0.0235(7)$ & $0.0205(7)$ & $-0.0007(6)$ & $0.0009(7)$ & $-0.0026(7)$ \\
C16 & $0.0422(11)$ & $0.0181(8)$ & $0.0407(10)$ & $-0.0045(7)$ & $-0.0063(9)$ & $-0.0034(7)$ \\
C17 & $0.0353(11)$ & $0.0302(10)$ & $0.0443(11)$ & $-0.0068(8)$ & $-0.0071(9)$ & $0.0026(9)$ \\
\hline
\end{tabular}

Geometric parameters $\left(\hat{A},{ }^{\circ}\right)$

\begin{tabular}{|c|c|c|c|}
\hline $\mathrm{O} 1-\mathrm{C} 15$ & $1.197(2)$ & $\mathrm{C} 8-\mathrm{H} 8$ & 0.9300 \\
\hline $\mathrm{O} 2-\mathrm{C} 15$ & $1.334(2)$ & $\mathrm{C} 9-\mathrm{C} 10$ & $1.427(2)$ \\
\hline $\mathrm{O} 2-\mathrm{C} 16$ & $1.465(2)$ & $\mathrm{C} 9-\mathrm{C} 14$ & $1.438(2)$ \\
\hline $\mathrm{C} 1-\mathrm{C} 14$ & $1.406(2)$ & $\mathrm{C} 10-\mathrm{C} 11$ & $1.360(3)$ \\
\hline $\mathrm{C} 1-\mathrm{C} 2$ & $1.409(2)$ & $\mathrm{C} 10-\mathrm{H} 10$ & 0.9300 \\
\hline $\mathrm{C} 1-\mathrm{C} 15$ & $1.495(2)$ & $\mathrm{C} 11-\mathrm{C} 12$ & $1.419(3)$ \\
\hline $\mathrm{C} 2-\mathrm{C} 3$ & $1.425(2)$ & C11-H11 & 0.9300 \\
\hline $\mathrm{C} 2-\mathrm{C} 7$ & $1.439(2)$ & $\mathrm{C} 12-\mathrm{C} 13$ & $1.360(3)$ \\
\hline $\mathrm{C} 3-\mathrm{C} 4$ & $1.367(2)$ & $\mathrm{C} 12-\mathrm{H} 12$ & 0.9300 \\
\hline $\mathrm{C} 3-\mathrm{H} 3$ & 0.9300 & $\mathrm{C} 13-\mathrm{C} 14$ & $1.433(2)$ \\
\hline $\mathrm{C} 4-\mathrm{C} 5$ & $1.416(3)$ & $\mathrm{C} 13-\mathrm{H} 13$ & 0.9300 \\
\hline $\mathrm{C} 4-\mathrm{H} 4$ & 0.9300 & $\mathrm{C} 16-\mathrm{C} 17$ & $1.492(3)$ \\
\hline $\mathrm{C} 5-\mathrm{C} 6$ & $1.357(3)$ & C16-H16A & 0.9700 \\
\hline $\mathrm{C} 5-\mathrm{H} 5$ & 0.9300 & $\mathrm{C} 16-\mathrm{H} 16 \mathrm{~B}$ & 0.9700 \\
\hline $\mathrm{C} 6-\mathrm{C} 7$ & $1.429(2)$ & C17-H17A & 0.9600 \\
\hline $\mathrm{C} 6-\mathrm{H} 6$ & 0.9300 & C17-H17B & 0.9600 \\
\hline $\mathrm{C} 7-\mathrm{C} 8$ & $1.397(2)$ & $\mathrm{C} 17-\mathrm{H} 17 \mathrm{C}$ & 0.9600 \\
\hline $\mathrm{C} 8-\mathrm{C} 9$ & $1.393(2)$ & & \\
\hline $\mathrm{C} 15-\mathrm{O} 2-\mathrm{C} 16$ & $117.96(15)$ & $\mathrm{C} 11-\mathrm{C} 10-\mathrm{H} 10$ & 119.4 \\
\hline $\mathrm{C} 14-\mathrm{C} 1-\mathrm{C} 2$ & $121.76(14)$ & $\mathrm{C} 9-\mathrm{C} 10-\mathrm{H} 10$ & 119.4 \\
\hline $\mathrm{C} 14-\mathrm{C} 1-\mathrm{C} 15$ & $118.97(15)$ & $\mathrm{C} 10-\mathrm{C} 11-\mathrm{C} 12$ & $120.39(17)$ \\
\hline $\mathrm{C} 2-\mathrm{C} 1-\mathrm{C} 15$ & $119.25(15)$ & $\mathrm{C} 10-\mathrm{C} 11-\mathrm{H} 11$ & 119.8 \\
\hline $\mathrm{C} 1-\mathrm{C} 2-\mathrm{C} 3$ & $122.97(14)$ & $\mathrm{C} 12-\mathrm{C} 11-\mathrm{H} 11$ & 119.8 \\
\hline $\mathrm{C} 1-\mathrm{C} 2-\mathrm{C} 7$ & $118.56(14)$ & $\mathrm{C} 13-\mathrm{C} 12-\mathrm{C} 11$ & $120.60(17)$ \\
\hline $\mathrm{C} 3-\mathrm{C} 2-\mathrm{C} 7$ & $118.46(14)$ & $\mathrm{C} 13-\mathrm{C} 12-\mathrm{H} 12$ & 119.7 \\
\hline $\mathrm{C} 4-\mathrm{C} 3-\mathrm{C} 2$ & $120.66(16)$ & $\mathrm{C} 11-\mathrm{C} 12-\mathrm{H} 12$ & 119.7 \\
\hline $\mathrm{C} 4-\mathrm{C} 3-\mathrm{H} 3$ & 119.7 & $\mathrm{C} 12-\mathrm{C} 13-\mathrm{C} 14$ & $121.09(16)$ \\
\hline $\mathrm{C} 2-\mathrm{C} 3-\mathrm{H} 3$ & 119.7 & $\mathrm{C} 12-\mathrm{C} 13-\mathrm{H} 13$ & 119.5 \\
\hline $\mathrm{C} 3-\mathrm{C} 4-\mathrm{C} 5$ & $120.90(17)$ & $\mathrm{C} 14-\mathrm{C} 13-\mathrm{H} 13$ & 119.5 \\
\hline $\mathrm{C} 3-\mathrm{C} 4-\mathrm{H} 4$ & 119.6 & $\mathrm{C} 1-\mathrm{C} 14-\mathrm{C} 13$ & $122.97(15)$ \\
\hline $\mathrm{C} 5-\mathrm{C} 4-\mathrm{H} 4$ & 119.6 & $\mathrm{C} 1-\mathrm{C} 14-\mathrm{C} 9$ & $118.88(14)$ \\
\hline $\mathrm{C} 6-\mathrm{C} 5-\mathrm{C} 4$ & $120.27(16)$ & $\mathrm{C} 13-\mathrm{C} 14-\mathrm{C} 9$ & $118.12(15)$ \\
\hline $\mathrm{C} 6-\mathrm{C} 5-\mathrm{H} 5$ & 119.9 & $\mathrm{O} 1-\mathrm{C} 15-\mathrm{O} 2$ & $124.50(15)$ \\
\hline
\end{tabular}




\begin{tabular}{|c|c|c|c|}
\hline $\mathrm{C} 4-\mathrm{C} 5-\mathrm{H} 5$ & 119.9 & $\mathrm{O} 1-\mathrm{C} 15-\mathrm{C} 1$ & $124.58(15)$ \\
\hline $\mathrm{C} 5-\mathrm{C} 6-\mathrm{C} 7$ & $121.11(16)$ & $\mathrm{O} 2-\mathrm{C} 15-\mathrm{C} 1$ & $110.92(14)$ \\
\hline $\mathrm{C} 5-\mathrm{C} 6-\mathrm{H} 6$ & 119.4 & $\mathrm{O} 2-\mathrm{C} 16-\mathrm{C} 17$ & $108.92(16)$ \\
\hline $\mathrm{C} 7-\mathrm{C} 6-\mathrm{H} 6$ & 119.4 & $\mathrm{O} 2-\mathrm{C} 16-\mathrm{H} 16 \mathrm{~A}$ & 109.9 \\
\hline $\mathrm{C} 8-\mathrm{C} 7-\mathrm{C} 6$ & $121.98(15)$ & $\mathrm{C} 17-\mathrm{C} 16-\mathrm{H} 16 \mathrm{~A}$ & 109.9 \\
\hline $\mathrm{C} 8-\mathrm{C} 7-\mathrm{C} 2$ & $119.44(14)$ & $\mathrm{O} 2-\mathrm{C} 16-\mathrm{H} 16 \mathrm{~B}$ & 109.9 \\
\hline $\mathrm{C} 6-\mathrm{C} 7-\mathrm{C} 2$ & $118.58(15)$ & $\mathrm{C} 17-\mathrm{C} 16-\mathrm{H} 16 \mathrm{~B}$ & 109.9 \\
\hline $\mathrm{C} 9-\mathrm{C} 8-\mathrm{C} 7$ & $121.98(15)$ & $\mathrm{H} 16 \mathrm{~A}-\mathrm{C} 16-\mathrm{H} 16 \mathrm{~B}$ & 108.3 \\
\hline $\mathrm{C} 9-\mathrm{C} 8-\mathrm{H} 8$ & 119.0 & $\mathrm{C} 16-\mathrm{C} 17-\mathrm{H} 17 \mathrm{~A}$ & 109.5 \\
\hline $\mathrm{C} 7-\mathrm{C} 8-\mathrm{H} 8$ & 119.0 & $\mathrm{C} 16-\mathrm{C} 17-\mathrm{H} 17 \mathrm{~B}$ & 109.5 \\
\hline $\mathrm{C} 8-\mathrm{C} 9-\mathrm{C} 10$ & $121.96(15)$ & $\mathrm{H} 17 \mathrm{~A}-\mathrm{C} 17-\mathrm{H} 17 \mathrm{~B}$ & 109.5 \\
\hline $\mathrm{C} 8-\mathrm{C} 9-\mathrm{C} 14$ & $119.34(15)$ & $\mathrm{C} 16-\mathrm{C} 17-\mathrm{H} 17 \mathrm{C}$ & 109.5 \\
\hline $\mathrm{C} 10-\mathrm{C} 9-\mathrm{C} 14$ & $118.69(15)$ & $\mathrm{H} 17 \mathrm{~A}-\mathrm{C} 17-\mathrm{H} 17 \mathrm{C}$ & 109.5 \\
\hline $\mathrm{C} 11-\mathrm{C} 10-\mathrm{C} 9$ & $121.11(16)$ & $\mathrm{H} 17 \mathrm{~B}-\mathrm{C} 17-\mathrm{H} 17 \mathrm{C}$ & 109.5 \\
\hline $\mathrm{C} 14-\mathrm{C} 1-\mathrm{C} 2-\mathrm{C} 3$ & $177.19(15)$ & $\mathrm{C} 9-\mathrm{C} 10-\mathrm{C} 11-\mathrm{C} 12$ & $0.6(3)$ \\
\hline $\mathrm{C} 15-\mathrm{C} 1-\mathrm{C} 2-\mathrm{C} 3$ & $-1.3(2)$ & $\mathrm{C} 10-\mathrm{C} 11-\mathrm{C} 12-\mathrm{C} 13$ & $-0.2(3)$ \\
\hline $\mathrm{C} 14-\mathrm{C} 1-\mathrm{C} 2-\mathrm{C} 7$ & $-1.8(2)$ & $\mathrm{C} 11-\mathrm{C} 12-\mathrm{C} 13-\mathrm{C} 14$ & $-0.3(3)$ \\
\hline $\mathrm{C} 15-\mathrm{C} 1-\mathrm{C} 2-\mathrm{C} 7$ & $179.65(14)$ & $\mathrm{C} 2-\mathrm{C} 1-\mathrm{C} 14-\mathrm{C} 13$ & $-179.90(15)$ \\
\hline $\mathrm{C} 1-\mathrm{C} 2-\mathrm{C} 3-\mathrm{C} 4$ & $-178.95(16)$ & $\mathrm{C} 15-\mathrm{C} 1-\mathrm{C} 14-\mathrm{C} 13$ & $-1.4(2)$ \\
\hline $\mathrm{C} 7-\mathrm{C} 2-\mathrm{C} 3-\mathrm{C} 4$ & $0.1(2)$ & $\mathrm{C} 2-\mathrm{C} 1-\mathrm{C} 14-\mathrm{C} 9$ & $2.0(2)$ \\
\hline $\mathrm{C} 2-\mathrm{C} 3-\mathrm{C} 4-\mathrm{C} 5$ & $-1.0(3)$ & $\mathrm{C} 15-\mathrm{C} 1-\mathrm{C} 14-\mathrm{C} 9$ & $-179.43(15)$ \\
\hline $\mathrm{C} 3-\mathrm{C} 4-\mathrm{C} 5-\mathrm{C} 6$ & $0.7(3)$ & $\mathrm{C} 12-\mathrm{C} 13-\mathrm{C} 14-\mathrm{C} 1$ & $-177.67(17)$ \\
\hline $\mathrm{C} 4-\mathrm{C} 5-\mathrm{C} 6-\mathrm{C} 7$ & $0.6(3)$ & $\mathrm{C} 12-\mathrm{C} 13-\mathrm{C} 14-\mathrm{C} 9$ & $0.4(3)$ \\
\hline $\mathrm{C} 5-\mathrm{C} 6-\mathrm{C} 7-\mathrm{C} 8$ & $178.35(17)$ & $\mathrm{C} 8-\mathrm{C} 9-\mathrm{C} 14-\mathrm{C} 1$ & $-0.8(2)$ \\
\hline $\mathrm{C} 5-\mathrm{C} 6-\mathrm{C} 7-\mathrm{C} 2$ & $-1.5(3)$ & $\mathrm{C} 10-\mathrm{C} 9-\mathrm{C} 14-\mathrm{C} 1$ & $178.09(15)$ \\
\hline $\mathrm{C} 1-\mathrm{C} 2-\mathrm{C} 7-\mathrm{C} 8$ & $0.3(2)$ & $\mathrm{C} 8-\mathrm{C} 9-\mathrm{C} 14-\mathrm{C} 13$ & $-178.94(15)$ \\
\hline $\mathrm{C} 3-\mathrm{C} 2-\mathrm{C} 7-\mathrm{C} 8$ & $-178.70(16)$ & $\mathrm{C} 10-\mathrm{C} 9-\mathrm{C} 14-\mathrm{C} 13$ & $-0.1(2)$ \\
\hline $\mathrm{C} 1-\mathrm{C} 2-\mathrm{C} 7-\mathrm{C} 6$ & $-179.79(16)$ & $\mathrm{C} 16-\mathrm{O} 2-\mathrm{C} 15-\mathrm{O} 1$ & $-4.4(3)$ \\
\hline $\mathrm{C} 3-\mathrm{C} 2-\mathrm{C} 7-\mathrm{C} 6$ & $1.2(2)$ & $\mathrm{C} 16-\mathrm{O} 2-\mathrm{C} 15-\mathrm{C} 1$ & $176.17(15)$ \\
\hline $\mathrm{C} 6-\mathrm{C} 7-\mathrm{C} 8-\mathrm{C} 9$ & $-178.99(16)$ & $\mathrm{C} 14-\mathrm{C} 1-\mathrm{C} 15-\mathrm{O} 1$ & $-74.0(2)$ \\
\hline $\mathrm{C} 2-\mathrm{C} 7-\mathrm{C} 8-\mathrm{C} 9$ & $0.9(3)$ & $\mathrm{C} 2-\mathrm{C} 1-\mathrm{C} 15-\mathrm{O} 1$ & $104.6(2)$ \\
\hline $\mathrm{C} 7-\mathrm{C} 8-\mathrm{C} 9-\mathrm{C} 10$ & $-179.49(16)$ & $\mathrm{C} 14-\mathrm{C} 1-\mathrm{C} 15-\mathrm{O} 2$ & $105.43(17)$ \\
\hline $\mathrm{C} 7-\mathrm{C} 8-\mathrm{C} 9-\mathrm{C} 14$ & $-0.7(3)$ & $\mathrm{C} 2-\mathrm{C} 1-\mathrm{C} 15-\mathrm{O} 2$ & $-76.00(19)$ \\
\hline $\mathrm{C} 8-\mathrm{C} 9-\mathrm{C} 10-\mathrm{C} 11$ & $178.42(18)$ & $\mathrm{C} 15-\mathrm{O} 2-\mathrm{C} 16-\mathrm{C} 17$ & $-108.52(18)$ \\
\hline $\mathrm{C} 14-\mathrm{C} 9-\mathrm{C} 10-\mathrm{C} 11$ & $-0.4(3)$ & & \\
\hline
\end{tabular}

Hydrogen-bond geometry $\left(\AA,{ }^{\circ}\right)$

\begin{tabular}{lllll}
\hline$D-\mathrm{H} \cdots A$ & $D-\mathrm{H}$ & $\mathrm{H} \cdots A$ & $D \cdots A$ & $D-\mathrm{H} \cdots A$ \\
\hline $\mathrm{C} 5-\mathrm{H} 5 \cdots \mathrm{O} 1^{\mathrm{i}}$ & 0.93 & 2.53 & $3.302(2)$ & 140 \\
\hline
\end{tabular}

Symmetry code: (i) $-x,-y+2, z+1 / 2$. 